\title{
Susceptibilidad de Cepas de Candida Oral a Extracto Etanólico del Propóleo Chileno de Olmué
}

\author{
Susceptibility of Oral Candida Strains to Ethanol Extract of Chilean Propolis of Olmué
}

\author{
Natalia Maureira'; Paulina Viera'; Alejandra Fernandez ${ }^{1,2,3}$; Madelein Urrejola ${ }^{1,2}$; \\ Cristian Bravo"; Francisca Mardones ${ }^{1}$; Enrique D. Vines ${ }^{4}$ \& Ziyad S. Haidar ${ }^{5,6,7}$
}

MAUREIRA, N.; VIERA, P.; FERNANDEZ, A.; URREJOLA, M.; BRAVO, C.; MARDONES, F.; VINES, E. D. \& HAIDAR, Z. S. Susceptibilidad de cepas de Candida oral a extracto etanólico del propóleo chileno de Olmué. Int. J. Odontostomat., 11(3):295-303, 2017.

RESUMEN: La infección por Candida albicans en la mucosa oral es conocida como Candidiasis oral (CO) y se diagnostica por el reconocimiento de cambios clínicos y la presencia de pseudohifas, hifas o levaduras en muestras obtenidas por citología exfoliativa o biopsia. Los agentes farmacológicos tópicos clásicos para el tratamiento de CO son Nistatina y Miconazol. Sin embargo, a pesar de las distintas terapias contra CO, existen formas de Candida resistentes al tratamiento convencional. El objetivo de este estudio fue determinar la susceptibilidad in vitro de Candida spp. a un extracto etanólico de propóleo de Olmué. Se realizó un estudio experimental descriptivo in vitro en donde se evaluó el efecto que presenta el uso de extracto etanólico de propóleo como antifúngico sobre cepas de Candida spp. obtenidas de la cavidad oral (mucosa palatina) de 31 individuos, con candidiasis oral diagnosticados con estomatitis subprotésica. El propóleo chileno utilizado fue obtenido de la zona geográfica de Olmué, quinta región. Se encontró que el $100 \%$ de las muestras en rangos de concentración de propóleo de $0,1 \mu \mathrm{g} / \mathrm{mL}$ y $1,6 \mu \mathrm{g} / \mathrm{mL}$ presentaron un grado de inhibición en el crecimiento de Candida Oral y por otra parte el extracto etanólico de propóleo que generó inhibición en la mayor cantidad de muestras fue al 0,4 $\mu \mathrm{g} / \mathrm{mL}$ $(41,94 \%$ de las muestras) y en segundo lugar la concentración al 0,2 $\mu \mathrm{g} / \mathrm{mL}$ (35 \% de las muestras). Se concluyó que el extracto etanólico de propóleo chileno obtenido de la zona de Olmué presenta la capacidad de inhibir el crecimiento de Candida spp. en agar Sabouraud in vitro de forma dosis dependiente.

PALABRAS CLAVE: Propóleo, Candida albicans, cavidad oral.

\section{INTRODUCCIÓN}

La Candida es un microorganismo oportunista clasificado dentro del grupo de levaduras, de los cuales se han identificado más de 150 especies. En los seres humanos es parte de la flora comensal de piel, tracto gastrointestinal y genitourinario, entre otras (Moosazadeh et al., 2016). Esta especie de levaduras es la más encontrada en la cavidad oral presentándose en el $80 \%$ de individuos sanos (Colombo et al., 2013), incluso su prevalencia aumenta en niños, adultos jóvenes y pacientes hospitalizados (Stoopler \& Sollecito, 2014). Esto se debe a que la Candida aumenta su proliferación cuando se pierde el equilibrio de las condiciones locales de la boca.
La infección por Candida albicans en la mucosa oral es conocida como Candidiasis oral (CO) y se diagnostica por el reconocimiento de cambios clínicos y la presencia de pseudohifas, hifas o levaduras en muestras obtenidas por citología exfoliativa o biopsia (Lewis et al., 2014). La CO se presenta principalmente como lesiones blancas o eritematosas. La CO de aspecto blanquecino incluye a la candidiasis pseudomembranosa y a la candidiasis hiperplásica crónica. La candidiasis de aspecto eritematosos incluye a la candidiasis atrófica crónica, candidiasis atrófica aguda, glositis romboidal media y queilitis an-

${ }^{1}$ Facultad de Odontología, Universidad Andrés Bello, Santiago, Chile.

2 BioMAT'X, Universidad de los Andes, Santiago, Chile.

${ }^{3}$ Programa de Doctorado en BioMedicina, Facultad de Medicina, Universidad de los Andes, Santiago, Chile.

${ }^{4}$ Facultad de Ciencias Biológicas, Departamento de Ciencias Biológicas, Universidad Andrés Bello, Santiago, Chile.

${ }^{5}$ Facultad de Odontología, Universidad de los Andes, Santiago, Chile.

${ }^{6}$ Plan de Mejoramiento Institucional (PMI) en Innovación I+D+i, Universidad de los Andes, Santiago, Chile.

${ }^{7}$ Centro de Investigación Biomédica, Universidad de los Andes, Santiago, Chile. 
gular. La candidiasis atrófica crónica es el tipo más frecuente de todas las $\mathrm{CO}$ y es conocida como candidiasis subprotésica, debido a que se presenta en la mucosa oral que se encuentra en contacto con la superficie de alguna prótesis removible. Clínicamente se observa un área eritematosa y se ubica en el paladar y reborde alveolar (Millsop et al., 2016). La clasificación de Newton descrita el año 1962, permite identificar el grado de estomatitis subprotésica, de acuerdo a 3 variedades clínicas (Ceballos Salobreña \& Delgado Azareño, 1996) (Fig. 1).

La candidiasis sub-protésica puede causar ardor oral y faríngeo, aumento de la sensibilidad interfiriendo en la alimentación, alteración del gusto y olfato, disfagia, odinofagia (Gall et al., 2013), aumento de volumen en la mucosa oral interfiriendo en la estética y en la estabilidad del uso de prótesis removible, conduciendo a alterar la calidad de vida de los individuos infectados (Moosazadeh et al.). Incluso, la presencia de $\mathrm{CO}$ se ha asociado a lesiones pontencialmente malignas y cáncer oral (Hamdy et al., 2016). Es por estos motivos que el tratamiento de la $\mathrm{CO}$ resulta imprescindible.

El tratamiento de la candidiasis oral consiste en el manejo de los factores predisponentes locales y

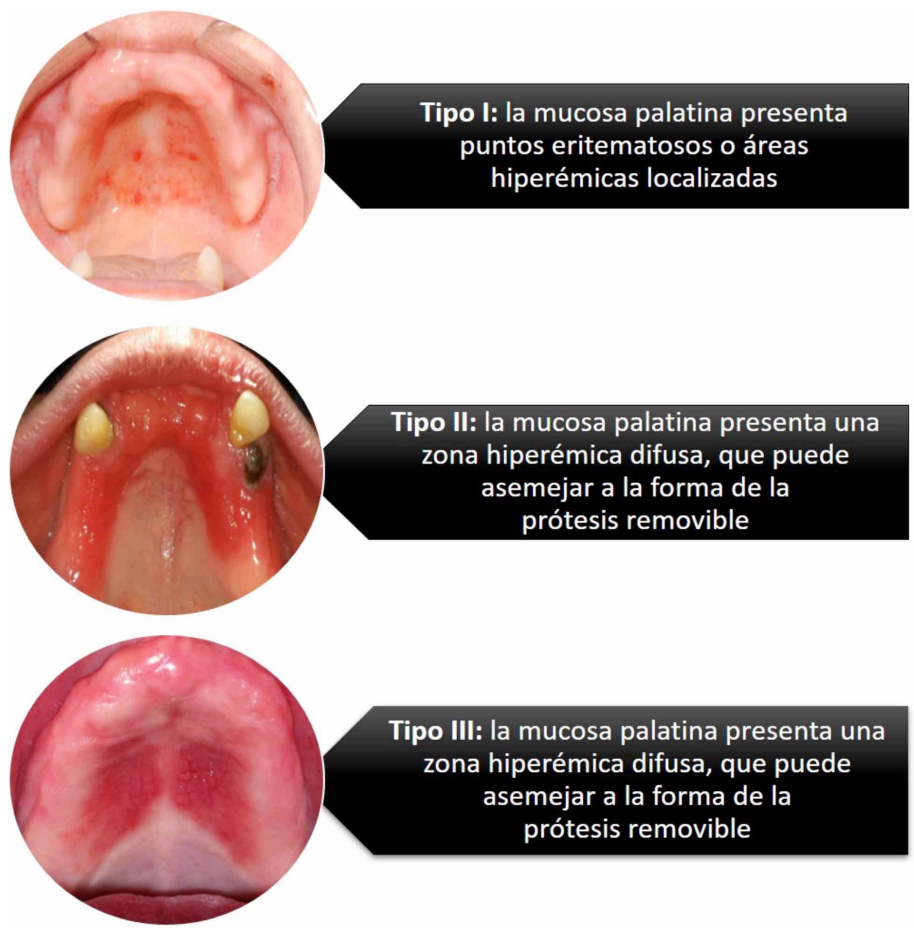

Fig. 1. Variedades clínicas de Newton (Imágenes - Servicio de Histopatología, Facultad de Odontología, Universidad Andrés Bello). sistémicos, en conjunto con el manejo farmacológico mediante agentes tópicos debido a que la infección por Cándida es de carácter superficial (Tobudic et al., 2012). Los agentes farmacológicos tópicos clásicos para el tratamiento de CO son Nistatina y Miconazol. Sin embargo, a pesar de las distintas terapias contra $\mathrm{CO}$, existen formas de Candida resistentes al tratamiento convencional, debido a la prescripción descontrolada de los antimicóticos (Martins et al., 2009). Es por tal motivo que se han propuesto alternativas terapéuticas naturales al tratamiento convencional (Garcia-Cuesta et al., 2014). La ventaja del uso de terapéuticos naturales en el tratamiento de la CO es que se encuentran disponibles en variados alimentos y plantas, por lo que su uso, adicionalmente estaría aportando con vitaminas y reduciendo los efectos tóxicos de los fármacos convencionales (Garcia-Cuesta et al.; Machorowska-Pieniazek et al., 2016).

En el área de la odontología el propóleo ha sido recomendado como terapia natural para mantener la higiene oral (Niedzielska et al., 2016), como antiséptico intracanal (Prabhakar et al., 2015), para desinfección cavitaria (AkhavanKarbassi et al., 2016), para el tratamiento de mucositis oral (Herrera et al., 2010), debido a que presenta propiedades antimicrobianas Figure 2. Sin embargo, a pesar de las investigaciones del uso del propóleo en la cavidad oral, se necesitan más estudios para su indicación en terapias orales (Espinoza et al., 2003).

El objetivo de este estudio fue determinar la susceptibilidad in vitro de Candida spp. a un extracto etanólico de propóleo de Olmué.

\section{MATERIAL Y MÉTODO}

Se diseñó un estudio experimental in vitro. El estudio fue aprobado por el comité de bioética, folio 037 año 2015, Facultad de Odontología, Universidad Andrés Bello. En la Figure 3 se observa el diagrama de flujo del experimento.

La población de interés correspondió a los pacientes de la Clínica Odontológica de la Facultad de Odontología de la Universidad Andrés Bello, sede Viña del Mar. La subpoblación de estudio fueron los individuos diagnosticados de Candidiasis Sub-protésica, entre mayo de 2014 y marzo de 2015. Los in- 


\section{Propóleo o Própolis}

Beneficios, Propiedades, Usos y Aplicaciones

¿es bueno para la salud?

Anti-microbianos

Anti-bacteriano

Anti-fúngicos

Anti-virulento

Bactericida / Bacteriostática

Cavidad dental y posterior extracción dental

Gingivitis

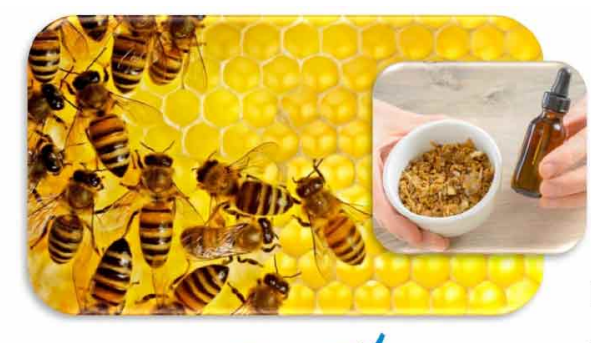

Trata enfermedades óseas e induce regeneración ósea Reduce el colesterol y la presión arterial

Trata alergias y intoxicación alimentaria

Mata las células cancerosas

Trata las verrugas y el herpes

Trata el estrés cardíaco

Antihistamínico

Hemostático
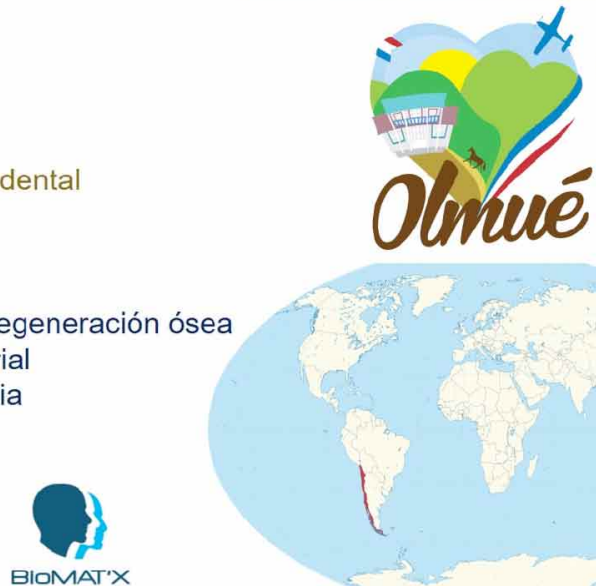

Fig. 2. Beneficios del propóleo.

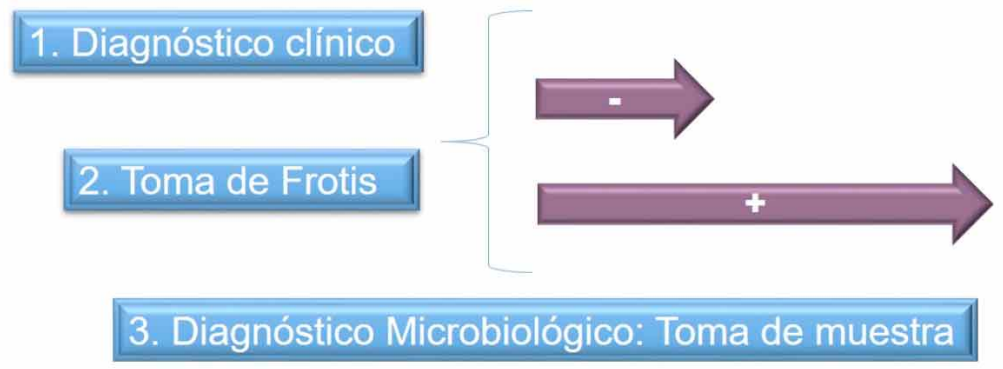

\section{Diagnóstico Microbiológico}
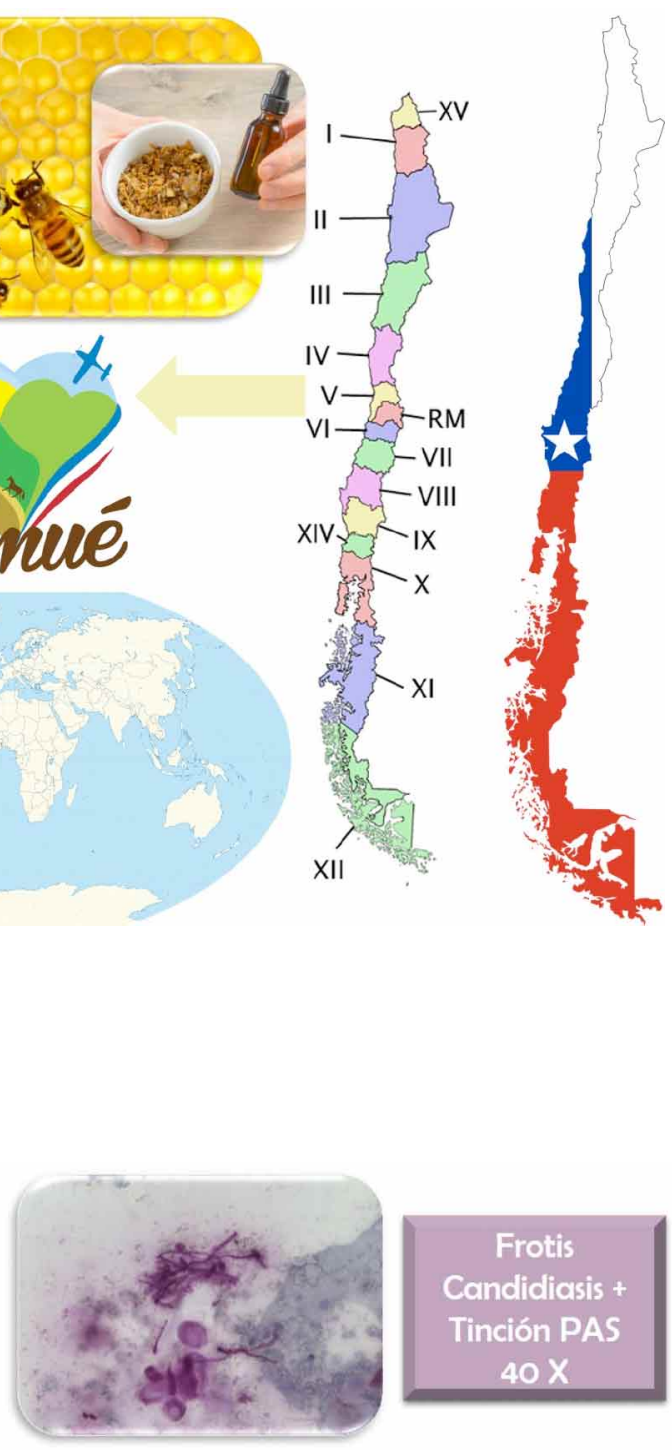

3. Diagnóstico Microbiológico: Toma de muestra

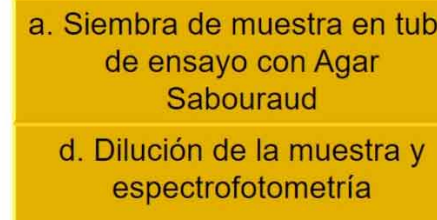

a. Siembra de muestra en tubo ensayo con Agar

ución de la muestra y espectrofotometría

\begin{tabular}{|c|}
\hline b. Incubar $48 \mathrm{hrs} / 37^{\circ} \mathrm{C}$ \\
e. Resiembra en placas de agar \\
Sabouraud y extracto etanólico de \\
propóleo al $0.025,0.05,0.1,0.2,0.4$ \\
$0.8,1.6,3.2 \%$ e incubar $\left(48 \mathrm{hrs} / 37^{\circ} \mathrm{C}\right)$
\end{tabular}

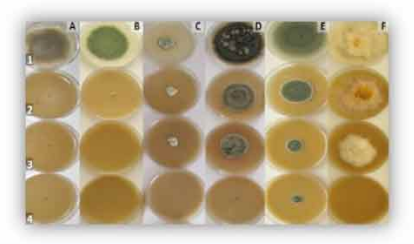

c. Observación microscópica de morfología de colonias con Tinción Gram

f. Observar placas, determinar MIC y registrar resultados

Fig. 3. Diagrama de flujo del Experimento. El diagrama de flujo indica los pasos para realizar el experimento. El diagnóstico de candidiasis oral se realiza principalmente a través de la observación clínica de las lesiones. A pesar de la estrecha relación entre la presencia de estomatitis sub-protésica y candidiasis palatina, es recomendable confirmar la presencia de Candida spp., para esto se realizan procedimientos de laboratorio como la observación microscópica de frotis o el cultivo de las muestras en medio Sabouraud (Williams \& Lewis, 2011). 
dividuos elegibles que cumplieron con los criterios de inclusión y que accedieron de forma voluntaria a participar, se les explicó el estudio de forma oral y escrita y se les entregó un consentimiento informado, el cual firmaron. Los criterios de inclusión correspondieron a individuos mayores de 18 años, portadores de prótesis removible superior parcial o total, diagnosticados clínicamente con estomatitis sub-protésico y positivos al frotis para Candida spp. Los criterios de exclusión correspondieron a individuos en terapia antimicótica tópica en la cavidad oral y sistémica, en terapia con colutorios antisépticos tales como clorhexidina al 0,12 $\%$, en individuos en tratamiento con corticoides tópicos como uso de inhalador con corticoides y mujeres embarazada y en periodo de lactancia.

Los individuos fueron seleccionados de manera aleatoria simple. De un total de 66 individuos con diagnóstico clínico y microbiológico de candidiasis subprotésica, se incluyeron 31 individuos.

Examen clínico. Dos patólogos orales, previamente calibrados, examinaron la mucosa oral de los individuos en un sillón dental (Unit 4T, Kavo, USA), bajo luz dental (A-dec 500, A-dec, USA), utilizando un espejo dental.

Toma de frotis oral. Los individuos con diagnóstico clínico de candidiasis subprotésica, se les tomó una muestra palatina para confirmar su diagnóstico con un frotis. Con una gasa se eliminó el exceso de saliva en el paladar y con una espátula de madera se raspó en una dirección la mucosa palatina. La muestra fue esparcida en un portaobjetos limpio y seco, 3 veces en la misma dirección abarcando distintas áreas. Se fijó con spray, y se envió la muestra al Servicio de Histopatología de la Facultad de Odontología, Universidad Andrés Bello, para su evaluación.

Toma de muestra. Se tomó muestra palatina de los individuos con diagnóstico clínico de candidiasis subprotésica y confirmada por el frotis, y que cumplieron con los criterios de inclusión y exclusión. La mucosa palatina fue limpiada con una gasa estéril y mediante una espátula de madera estéril se raspó la zona de la lesión hasta observar una muestra visible en la punta de la espátula. Se retiró el tapón de algodón del tubo de ensayo y se flameó el borde de éste bajo un mechero. Se esparció mediante la técnica de siembra en estría sobre el tubo de ensayo con agar Sabouraud tendido estéril. Se flameó nuevamente el borde del tubo de ensayo bajo el mechero y se colocó el tapón de algodón. La muestra fue en- viada al Laboratorio de Microbiología de la Universidad Andrés Bello sede Viña del Mar.

\section{Preparación del extracto etanólico de propóleo} al $30 \%$. El propóleo chileno utilizado en la investigación fue obtenido de la zona geográfica de Olmué, en la región de Valparaíso, Chile. El período en el que fue extraído correspondió al verano del año 2014. Para la obtención del propóleo puro se mezclaron 300 gramos de propóleo con 1 litro de alcohol etílico potable al $96 \%$ y esta mezcla se maceró por 3 semanas, a temperatura ambiente (entre 20 a $30^{\circ} \mathrm{C}$ ) revolviendo el preparado 1 vez al día. Se filtró con mallas de nylon de 100 mesh para eliminar las impurezas del preparado. Una vez completado este proceso se obtuvo el extracto etanólico de propóleo a concentración del $30 \%$.

Manipulación de las muestras. Se realizó la resiembra de la muestra la cual se incubó a $37^{\circ} \mathrm{C}$ por 48 horas. Una vez que creció la resiembra, se observó si macroscópicamente con la morfología de colonias de Candida spp. se tomó con un asa de punta redonda una colonia, la cual se extendió sobre un portaobjetos para realizar una tinción de Gram y se observó en el microscopio la presencia de levaduras para confirmar que las colonias correspondan a Candida spp. (Fig. 3).

Se tomó con un asa redonda una porción de la muestra la que se diluyó en $5 \mathrm{~mL}$ de cloruro de sodio al 0,09\% estéril en un tubo de ensayo estéril, se extrajeron $100 \mu \mathrm{L}$ de esta suspensión la cual se colocó en una cubetilla con $900 \mu \mathrm{L}$ de cloruro de sodio al 0,09\%, se colocó en el espectrofotómetro para la lectura de la muestra. A modo de estandarizar la cantidad de microorganismos en cada placa se utilizó una absorbancia de 200, es decir, una vez que se obtuvo la absorbancia de la cubetilla se diluyó la muestra que se encontró en el tubo, la cantidad necesaria para alcanzar la absorbancia establecida. Se colocó $50 \mu \mathrm{L}$ de la suspensión en cada placa y mediante la técnica de rastrillo de con un asa de vidrio se sembró sobre la superficie del agar hasta que la suspensión se secó. Se incubó las placas con agar Sabouraud y extracto etanólico de propóleo al 0,025, $0,05,0,1,0,2,0,4,0,8,1,6 \%$, por 48 horas a $37^{\circ} \mathrm{C}$, se observaron las placas para observar si hubo crecimiento, para determinar la concentración inhibitoria mínima Candida spp., en los casos en que hubo crecimiento, en todas las placas se repitió el experimento incluyendo 2 concentraciones extra al 1,6 y 3,2\% (Fig. 3). 
Variables en estudio. Sexo (masculino-femenino), edad (en años), tipo de prótesis removible (total, parcial), material de la prótesis removivble (acrílica, metálica), clasificación de Newton $(1,2,3)$, tiempo de usos de prótesis (años) crecimiento de Candida spp. en el agar (si muestra crecimiento-no muestra crecimiento), concentración inhibitoria mínima propóleo (CIM) $(\mu \mathrm{g} / \mathrm{mL})$.

Análisis estadístico. Las variables cualitativas (género y crecimiento de Candida spp. en el agar) se representaron mediante frecuencia absoluta y relativa. Las variables continuas (edad, CIM) se representaron mediante media y desviación estándar. La relación de las variables fue mediante test chi cuadrado de Pearson y test de Fisher. Todos los cálculos fueron realizados en BioMAT'X - CIB, Universidad de los Andes, Santiago de Chile, con el Stats Direct Statistical software versión 2.8.0.

\section{RESULTADOS}

De un total de 66 pacientes diagnosticados con candidiasis crónica eritematosa asociado a estomatitis sub-protésica, 46 pacientes fueron mujeres $(69,70$ $\%)$ y 20 hombres (30,30\%), en donde se seleccionaron 31 pacientes que cumplieron con los criterios de inclusión y exclusión, 21 eran mujeres $(67,74 \%)$ y 10 hombres $(32,26 \%)$.

De los 31 pacientes que participaron del estudio, su distribución según edad abarca un rango etario entre los 26 y 81 años. El promedio de edad de la muestra fue de 61,84 años.

Con respecto a la clasificación de Newton para las candidiasis sub-protésicas de acuerdo a su presentación clínica se observó el mayor porcentaje en el tipo I, el cual se manifestó en 17 pacientes (54,84 \%) (Tabla I).

Tabla I. Clasificación de Newton.

\begin{tabular}{ccl}
\hline Clasificación de Newton & Frecuencia & Porcentaje \\
\hline I & 17 & $54,84 \%$ \\
II & 8 & $25,81 \%$ \\
III & 6 & $19,35 \%$ \\
Total & 31 & $100,00 \%$ \\
\hline
\end{tabular}

Los resultados de presencia o ausencia de crecimiento de colonias de Candida spp. se observan en la Tabla II.
Tabla II. Presencia o ausencia de crecimiento de colonias de Candida spp.

\begin{tabular}{ccc}
\hline $\mathbf{C I M}$ & $\mathbf{S i}$ & No \\
\hline 0 & 31 & 0 \\
0,025 & 31 & 0 \\
0,05 & 31 & 0 \\
0,1 & 27 & 4 \\
0,2 & 16 & 15 \\
0,4 & 3 & 28 \\
0,8 & 2 & 29 \\
1,6 & 0 & 31 \\
3,2 & 0 & 31 \\
\hline
\end{tabular}

Con respecto a la concentración mínima inhibitoria, se pudo confirmar que el $100 \%$ de las muestras en rangos de concentración de propóleo de $0,1 \mu \mathrm{g} / \mathrm{mL}$ y $1,6 \mu \mathrm{g} / \mathrm{mL}$ presentaron inhibición en su crecimiento. Mientras que en todas las muestras con concentraciones de 0,025 y $0,05 \mu \mathrm{g} / \mathrm{mL}$ hubo crecimiento de la levadura, ver Tabla III. Por otra parte, se observó que la concentración de extracto etanólico de propóleo que generó inhibición en la mayor cantidad de muestras fue al $0,4 \mu \mathrm{g} / \mathrm{mL}(41,94 \%$ de las muestras) y en segundo lugar la concentración al $0,2 \mu \mathrm{g} / \mathrm{mL}$ (35\% de las muestras). Cabe destacar que entre las muestras con concentración de $0,1 \mu \mathrm{g} / \mathrm{mL}$ y $0,4 \mu \mathrm{g} /$ $\mathrm{mL}$, ya el $90,32 \%$ del total de las muestras se encontraba con inhibición del crecimiento de Candida spp. (Tabla III).

Tabla III. Concentración inhibitoria mínima.

\begin{tabular}{ccccc}
\hline $\begin{array}{c}\mathrm{MIC} \\
\mu \mathrm{g} / \mathrm{mL}\end{array}$ & $\begin{array}{c}\text { Frecuencia } \\
\text { de } \\
\text { inhibición }\end{array}$ & $\begin{array}{c}\text { Frecuencia } \\
\text { acumulada }\end{array}$ & $\%$ & $\begin{array}{c}\% \\
\text { acumulado }\end{array}$ \\
\hline 0,1 & 4 & 4 & 12,90 & 12,90 \\
0,2 & 11 & 15 & 35,48 & 48,38 \\
0,4 & 13 & 28 & 41,94 & 90,32 \\
0,8 & 1 & 29 & 3,23 & 93,55 \\
1,6 & 2 & 31 & 6,45 & 100,00 \\
Total & 31 & 31 & 100,00 & 100,00 \\
\hline
\end{tabular}

Respecto a la relación entre la presentación clínica de la candidiasis oral a través de la clasificación de Newton y la concentración mínima inhibitoria, se realizó el test estadístico de chi cuadrado de Pearson, en el cual se obtuvo como resultado un p valor de 0,059 y el de Fisher un $r$ valor de 0,059 , es decir, se considera que ambas variables son independientes (Tabla IV).

La relación entre el género y la concentración inhibitoria mínima se realizó el test estadístico de chi cuadrado de Pearson de lo que se obtuvo un $r$ valor de 0,071 y en el test de Fisher el resultado del $r$ valor 
Tabla IV. Descripción entre clasificación de Newton y MIC.

\begin{tabular}{ccccccccc}
\hline MIC & $\begin{array}{c}\text { Newton } \\
\mathrm{n} 1\end{array}$ & $\%$ & $\begin{array}{c}\text { Newton } \\
\mathrm{n} 2\end{array}$ & $\%$ & $\begin{array}{c}\text { Newton } \\
\mathrm{n} 3\end{array}$ & $\%$ & Total & $\%$ \\
\hline 0,1 & 2 & 6,45 & 1 & 3,23 & 1 & 3,23 & 4 & 12,90 \\
0,2 & 6 & 19,35 & 2 & 6,45 & 3 & 9,68 & 11 & 35,48 \\
0,4 & 9 & 29,03 & 4 & 12,90 & 0 & 0,00 & 13 & 41,94 \\
0,8 & 0 & 0,00 & 1 & 3,23 & 0 & 0,00 & 1 & 3,23 \\
1,6 & 0 & 0,00 & 0 & 0,00 & 2 & 6,45 & 2 & 6,45 \\
Total & 17 & 54,84 & 8 & 25,81 & 6 & 19,35 & 31 & 100,0 \\
\hline
\end{tabular}

fue de 0,052 , es decir, las variables son independientes entre sí (Tabla V). Cabe destacar que de los pacientes involucrados en la investigación hubo una relación 2:1, del género femenino y masculino respectivamente. Estos resultados se relacionan con la proporción obtenida de los pacientes que, acudieron a la Universidad Andrés Bello sede Viña del Mar y que no fueron incluidos en el estudio.

Tabla V. Relación entre el sexo y MIC.

\begin{tabular}{cccc}
\hline MIC & Femenino & Masculino & Total \\
\hline 0,1 & 3 & 1 & 4 \\
0,2 & 10 & 1 & 11 \\
0,4 & 7 & 6 & 13 \\
0,8 & 1 & 0 & 1 \\
1,6 & 0 & 2 & 2 \\
TOTAL & 21 & 10 & 31 \\
\hline
\end{tabular}

Con respecto a la relación entre la edad y la concentración inhibitoria mínima se realizó el test estadístico de chi cuadrado de Pearson de lo que se obtuvo un valor de 8,46 , se obtuvo un r valor de 0,7234 $y$ al test de Fisher el $r$ valor fue de 0,7234; es decir, las variables son independientes entre sí (Tabla VI).

Tabla VI. Relación entre la edad y MIC.

\begin{tabular}{cccc}
\hline Concentración & Frecuencia & Promedio & $\begin{array}{c}\text { Desviación } \\
\text { estándar }\end{array}$ \\
\hline 0,1 & 4 & 65,0 & 12,62 \\
0,2 & 11 & 54,9 & 17,01 \\
0,4 & 13 & 65,9 & 8,26 \\
0,8 & 1 & 54 & 0 \\
1,6 & 2 & 71 & 2,83 \\
TOTAL & 31 & & \\
\hline
\end{tabular}

En base a la información obtenida respecto a la asociación entre los años de uso de la prótesis removible de los pacientes involucrados en el estudio y la concentración inhibitoria mínima obtenida se realizó el test estadístico de chi cuadrado de Pearson de lo que se obtuvo un valor de 24,46 y un $r$ valor de 0,8726 y en el test de Fisher se obtuvo un $r$ valor de
0,670; es decir, las variables son independientes entre sí (Tabla VII).

Tabla VII. Relación entre los años de uso de prótesis removible y MIC.

\begin{tabular}{cccc}
\hline Concentración & Frecuencia & Promedio & $\begin{array}{c}\text { Desviación } \\
\text { estándar }\end{array}$ \\
\hline 0,1 & 4 & 5,65 & 9,60 \\
0,2 & 11 & 8,38 & 6,96 \\
0,4 & 13 & 10,56 & 8,37 \\
0,8 & 1 & 25 & 0,00 \\
1,6 & 2 & 17,50 & 17,68 \\
TOTAL & 31 & & \\
\hline
\end{tabular}

De acuerdo al tipo de prótesis removible usada por los pacientes incluidos en la investigación, cuya categoría se dividió en prótesis parcial o total, y la concentración inhibitoria mínima se realizó el test estadístico de chi cuadrado de Pearson de lo que se obtuvo un valor de 7,259 y un $r$ valor de 0,122 , al realizar el test de Fisher el $r$ valor obtenido fue de 0,091, es decir, las variables son independientes entre sí (Tabla VIII).

Tabla VIII. Relación entre el tipo de prótesis removible y MIC.

\begin{tabular}{cccc}
\hline Concentración & Total & Parcial & Total \\
\hline 0,1 & 4 & 0 & 4 \\
0,2 & 6 & 5 & 11 \\
0,4 & 12 & 1 & 13 \\
0,8 & 1 & 0 & 1 \\
1,6 & 1 & 1 & 2 \\
TOTAL & 24 & 7 & 31 \\
\hline
\end{tabular}

Al estudiar la relación entre el material de la prótesis y la concentración inhibitoria mínima se realizó el test estadístico de chi cuadrado de Pearson que obtuvo un valor de 5,4611 y el r valor de 0,243, al realizar el test de Fisher el $r$ valor obtenido fue de 0,244, es decir, las variables son independientes entre sí (Tabla IX). 
Tabla IX. Relación entre el material de prótesis removible y MIC.

\begin{tabular}{cccc}
\hline Concentración & Acrílica & Metálica & Total \\
\hline 0,1 & 3 & 1 & 4 \\
0,2 & 10 & 1 & 11 \\
0,4 & 7 & 6 & 13 \\
0,8 & 1 & 0 & 1 \\
1,6 & 2 & 0 & 2 \\
TOTAL & 23 & 8 & 31
\end{tabular}

\section{DISCUSIÓN}

El presente estudio permitió demostrar que la Candida spp. obtenida del paladar de pacientes diagnosticados clínica y microbiológicamente con candidiasis eritematosa crónica sub-protésica, fueron susceptibles al extracto etanólico de propóleo obtenido de la zona de Olmué. Las muestras de Candida spp. fueron obtenidas principalmente de mujeres, en un rango entre los 50 y 60 años. Esto se puede deber a que generalmente son las mujeres las que más asisten a servicios de diagnósticos odontológicos (AL-Waili et al., 2012; Lynge Pedersen et al., 2015) y a que la mayor prevalencia de Candidiasis sub-protésica es en individuos sobre 50 años (AL-Waili et al.).

El rango de concentración inhibitoria mínima alcanzado para el propóleo obtenido de Olmué, contra Candida spp., en placas Petri fue de 0,1 a 1,6 $\mu \mathrm{g} /$ $\mathrm{mL}$. Rangos en el que coincidimos con AL-Waili et al., Kacániová et al. (2012) y Herrera et al.. Este resultado se puede deber a que a pesar de que los diferentes investigadores utilizamos propóleo de distintas zonas geográficas las concentraciones de flavonoides fueron similares y componente esencial en la actividad antifúngica del propóleo (Herrera et al.; AL-Waili et al.; Kacániová et al.). Específicamente, Herrera et al. determinaron la CIM en cepas de Candida spp. con propóleo de origen brasileño recolectado por abejas Apis mellifera de diferentes regiones del Brasil (sur y noreste de Brasil). AL-Waili et al. utilizaron propóleo recogido de Arabia Saudita (EEPS) y de Egipto (EEPE), determinaron la CIM cepas de Candida Albicans del Departamento de Microbiología, Unidad de Investigación de Abejas, Universidad King Saud, Riyadh. Kacániová et al. utilizaron cepas, aislados clínicos, que fueron suministrados por el hospital universitario de Martin (República de Eslovaquia) y el propóleo fue extraído de 2 localidades de la parte central de Eslovaquia (región de Detva).
Sin embargo, existen reportes en la literatura tales como Quintero-Mora et al. (2008), Haghdoost et al. (2016) y Tobaldini-Valerio et al. (2016) que difieren de nuestros resultados, ya que obtuvieron rangos de inhibición de entre 0,6 a $10 \mu \mathrm{g} / \mathrm{mL}, 220$ a 880 $\mu \mathrm{g} / \mathrm{mL}$ y $36 \mu \mathrm{g} / \mathrm{mL}$ a $125 \mu \mathrm{g} / \mathrm{mL}$, respectivamente. Esto podría deberse a que existieron diferencias en la preparación del propóleo y en el procedimiento de laboratorio, encontrándose distintas temperaturas, tiempo de incubación y concentración del etanol utilizado.

Existe una relación directa entre el tiempo de uso de una prótesis removible y la aparición de candidiasis. El criterio de Espinoza et al. indica que este fenómeno se presenta luego de 5 años de uso, tiempo que favorece el deterioro protésico y la colonización por Candida Spp. Es necesario tener presente que también influye en la aparición de la infección el desajuste protésico, los malos hábitos de higiene oral y períodos de baja defensa orgánica, que a medida que transcurre el tiempo hace más probable la aparición de candidiasis (Farah et al., 2011).

Existe una relación directa entre el tiempo de uso de una prótesis removible y la aparición de candidiasis. El criterio de Espinoza et al. (Espinoza et al, 2003); indica que este fenómeno se presenta luego de 5 años de uso, tiempo que favorece el deterioro protésico y la colonización por Cándida Spp. Es necesario tener presente que también influye en la aparición de la infección el desajuste protésico, los malos hábitos de higiene oral y períodos de baja defensa orgánica, que a medida que transcurre el tiempo hace más probable la aparición de candidiasis (Farah et al.).

Ahora bien, al realizar el vínculo de MIC con la clasificación clínica de Newton que aparece representado en la Tabla IV, la inhibición comenzara a los 0,1 $\mathrm{mg} / \mathrm{mL}$ para todos los tipos de Newton, y en la etapa 1 se registrara el menor nivel de concentración para la inhibición total de las muestras a $0,4 \mathrm{mg} / \mathrm{mL}$, secuencialmente seguido por tipo 2 con un $0,8 \mathrm{mg} / \mathrm{mL}$ y tipo 3 que requirió un valor de $1,6 \mathrm{mg} / \mathrm{mL}$. Pese a que no se encontró esta asociación en la literatura, los resultados dan un indicio que, a mayor intensidad del proceso patológico, mayor es la cantidad de propóleo que se debe usar, ya sea en la concentración o en el tiempo de aplicación. Los valores de concentración inhibitoria mínima de extracto etanólico de propóleo necesario para los tres tipos de diagnósticos clínicos de candidiasis estuvieron bajo los niveles de 
toxicidad establecidos por la European Food Safety Authority en el consenso del año 2010, cuyos niveles se definieron a los $2 \mathrm{~g} / \mathrm{Kg} /$ día.

Los resultados del presente estudio junto con el resultado del uso del propóleo de otros países (ALWaili et al.; Kacániová et al.), permiten indicar que el propóleo presenta propiedades antifúngicas capaz de inhibir el crecimiento de Candida spp. in vitro (Casaroto \& Lara, 2010).

Dentro de las limitaciones de esta investigación se encuentra la imposibilidad de determinar con exactitud la presencia y proporción de los componentes activos del propóleo. Dentro de los componentes activos, se vincula la concentración de flavonoides con la estandarización de este antifúngico, es importante señalar que en la literatura consultada se aclara que al margen de la composición de los diferentes tipos de propóleo, a nivel mundial se mantienen las propiedades fungicidas (Casaroto \& Lara; Popova et al., 2013).

\section{CONCLUSIÓN}

Se concluye que el extracto etanólico de propóleo chileno obtenido de la zona de Olmué presenta la capacidad de inhibir el crecimiento de Candida spp. en agar Sabouraud in vitro de forma dosis dependiente, alcanzado una acción fungicida de un $100 \%$.

MAUREIRA, N.; VIERA, P.; FERNANDEZ, A.; URREJOLA, M.; BRAVO, C.; MARDONES, F.; VINES, E. D. \& HAIDAR, Z. S. Susceptibility of oral Candida strains to ethanol extract of Chilean propolis of Olmué. Int. J. Odontostomat., 11(3):295-303, 2017.

ABSTRACT: Fungal (or yeast) infections; mycoses, occurring in the oral mucous membranes, of Candida species (mostly C. albicans, a normal component of the oral microbiota), also known as oral thrush or oral candidiasis $(\mathrm{OC})$, can be diagnosed via the recognition of clinical changes and the presence of pseudohyphae, hyphae or yeasts in samples obtained by exfoliative cytology and/or biopsy. Topical pharmacological preparations and drugs such as Nystatin and Miconazole are used in the treatment of $\mathrm{CO}$. However, there are forms of Candida with resistance to such conventional treatment approaches. Therefore, the aim of this study is to determine the in vitro susceptibility of Candida spp.; an ethanolic extract of propolis from Olmué. Hence, an experimental in vitro descriptive study was carried out in which the effect of an ethanolic extract of propolis used as antifungal on strains of Candida spp. obtained from the oral cavity (palatine mucosa) of 31 individuals, diagnosed with OC (subdenture stomatitis) is determined. Natural propolis was obtained from the Olmué area, in the 5th region of Chile. It was found that $100 \%$ of the samples with propolis concentration ranging from $0.1 \mathrm{mg} / \mathrm{mL}$ to $1.6 \mathrm{mg} / \mathrm{mL}$ presented a degree of inhibition in the growth of OC. On the other hand, the ethanolic extract of propolis that generated inhibition in the largestnumber of samples was $0.4 \mathrm{mg} / \mathrm{mL}$ ( $41.94 \%$ of the samples) followed by the concentration of $0.2 \mathrm{mg} / \mathrm{mL}$ (35\% of the samples). Therefore, it can be concluded that the ethanolic extract of Chilean propolis obtained from the Olmué area has the ability to inhibit the growth of Candida spp. in vitro in a dosage-dependent manner.

KEY WORDS: propolis, Candida albicans, oral cavity.

\section{REFERENCIAS BIBLIOGRÁFICAS}

AkhavanKarbassi, M.; Yazdi, M. F.; Ahadian, H. \& SadrAbad, M. J. Randomized doubleblind placebocontrolled trial of propolis for oral mucositis in patients receiving chemotherapy for head and neck cancer. Asian Pac. J. Cancer Prev., 17(7):3611-4, 2016.

AL-Waili, N.; Al-Ghamdi, A.; Ansari, M. J.; Al-Attal, Y. \& Salom, K. Synergistic effects of honey and propolis toward drug multiresistant Staphylococcus Aureus, Escherichia Coli and Candida Albicans isolates in single and polymicrobial cultures. Int. J. Med. Sci., 9(9):793-800, 2012.

Casaroto, A. R. \& Lara, V. S. Phytomedicines for Candida-associated denture stomatitis. Fitoterapia, 81(5):323-8, 2010.

Ceballos Salobreña, A. \& Delgado Azareño, W. Micosis Bucales. Madrid, Grupo Aula Médica, 1996. pp.144-8.

Colombo, A. L.; Guimarães, T.; Camargo, L. F.; Richtmann, R.; Queiroz-Telles, F. D.; Salles, M. J.; Cunha, C. A.; Yasuda, M. A.; Moretti, M. L. \& Nucci, M. Brazilian guidelines for the management of candidiasis - a joint meeting report of three medical societies: Sociedade Brasileira de Infectologia, Sociedade Paulista de Infectologia and Sociedade Brasileira de Medicina Tropical. Braz. J. Infect. Dis., 17(3):283-312, 2013.

Espinoza, I.; Rojas, R.; Aranda, W. \& Gamonal, J. Prevalence of oral mucosal lesions in elderly people in Santiago, Chile. J. Oral Pathol. Med., 32(10):571-5, 2003.

Farah, C. S.; Lynch, N. \& McCullough, M. J. Oral fungal infections: an update for the general practitioner. Aust. Dent. J., 55 Suppl. $1: 48-54,2010$.

Gall, F.; Colella, G.; Di Onofrio, V.; Rossiello, R.; Angelillo, I. F. \& Liguori, G. Candida spp. in oral cancer and oral precancerous lesions. New Microbiol., 36(3):283-8, 2013.

Garcia-Cuesta, C.; Sarrion-Pérez, M. G. \& Bagán, J. V. Current treatment of oral candidiasis: A literature review. J. Clin. Exp. Dent., 6(5):e576-82, 2014.

Haghdoost, N.; Salehi, T. Z.; Khosravi, A. \& Sharifzadeh, A. Antifungal activity and influence of propolis against germ tube formation as a critical virulence attribute by clinical isolates of Candida albicans. J. Mycol. Med., 26(4):298-305, 2016.

Hamdy, R. F.; Zaoutis, T. E. \& Seo, S. K. Antifungal stewardship considerations for adults and pediatrics. Virulence, 2:1-15, 2016.

Herrera, C. L.; Alvear, M.; Barrientos, L.; Montenegro, G. \& Salazar, L. The antifungal effect of six commercial extracts of Chilean propolis on Candida spp. Cienc. Investig. Agrar., 37(1):75-84, 2010. 
MAUREIRA, N.; VIERA, P.; FERNANDEZ, A.; URREJOLA, M.; BRAVo, C.; MARDONES, F.; VINES, E. D. \& HAIDAR, Z. S. Susceptibilidad de cepas de Candida oral a extracto etanólico del propóleo chileno de Olmué. Int. J. Odontostomat., 11(3):295-303, 2017.

Kacániová, M.; Vukovic, N.; Chlebo, R.; Hascík, P.; Rovná, K.; Cubon, J.; Dzugan, M. \& Pasternakiewicz, A. The antimicrobial activity of honey, bee pollen loads and beeswax from Slovakia. Arch. Biol. Sci., 64(3):927-34, 2012.

Lewis, M. The role of antifungal and antiviral agents in primary dental care. Prim. Dent. J., 3(4):59-64, 2014.

Lynge Pedersen, A. M.; Nauntofte, B.; Smidt, D. \& Torpet, L. A. Oral mucosal lesions in older people: relation to salivary secretion, systemic diseases and medications. Oral Dis. 21(6):721-9, 2015.

Machorowska-Pieniazek, A.; Skucha-Nowak, M.; Mertas, A.; Tanasiewicz, M.; Niedzielska, I.; Morawiec, T. \& Baron, S. Effects of brazilian propolis on dental plaque and gingiva in patients with oral cleft malformation treated with multibracket and removable appliances: A comparative study. Evid. Based Complement. Altern. Med., 2016: 2038407, 2016.

Martins, C. V.; da Silva, D. L.; Neres, A. T.; Magalhães, T. F.; Watanabe, G. A.; Modolo, L. V.; Sabino, A. A.; de Fátima, A. \& de Resende, M. A. Curcumin as a promising antifungal of clinical interest. J. Antimicrob. Chemother., 63(2):337-9, 2009.

Millsop, J. W. \& Fazel, N. Oral candidiasis. Clin. Dermatol., 34(4):48794, 2016.

Moosazadeh, M.; Akbari, M.; Tabrizi, R.; Ghorbani, A.; Golkari, A. \& Banakar, M. Denture stomatitis and Candida Albicans in Iranian population: A systematic review and meta-analysis. J. Dent. (Shiraz), 17(3 Suppl.):283-92, 2016.

Niedzielska, I.; Puszczewicz, Z.; Mertas, A.; Niedzielski, D.; Rózanowski, B.; Baron, S.; Konopka, T.; Machorowska-Pieniazek, A.; Skucha-Nowak, M.; Tanasiewicz, M.; Paluch, J.; Markowski, J.; Orzechowska-Wylegaa, B.; Król, W. \& Morawiec, T. The influence of ethanolic extract of Brazilian green propolis gel on hygiene and oral microbiota in patients after mandible fractures. Biomed. Res. Int., 2016:9190814, 2016.

Popova, M.; Dimitrova, R.; Al-Lawati, H.; Tsvetkova, I.; Najdenski, H. \& Bankova, V. Omani propolis: chemical profiling, antibacterial activity and new propolis plant sources. Chem. Cent. J., 7(1):158, 2013.

Prabhakar, A. Karuna, Y. M.; Yavagal, C. \& Deepak, B. M. Cavity disinfection in minimally invasive dentistry - comparative evaluation of Aloe vera and propolis: A randomized clinical trial. Contemp. Clin. Dent., 6(Suppl. 1):S24-31, 2015.

Quintero-Mora, M. L.; Londoño-Orozco, A.; Hernández-Hernández, F.; Manzano-Gayosso, P.; López-Martínez, R.; Soto-Zárate, C. I.; Carrillo-Miranda, L.; Penieres-Carrillo, G.; García-Tovar, C. G. \& Cruz-Sánchez, T. A. Effect of Mexican propolis extracts from Apis mellifera on Candida albicans in vitro growth. Rev. Iberoam. Micol., 25(1):22-6, 2008.

Stoopler, E. T. \& Sollecito, T. P. Oral mucosal diseases: evaluation and management. Med. Clin. North Am., 98(6):1323-52, 2014.

Tobaldini-Valerio, F. K.; Bonfim-Mendonça, P. S.; Rosseto, H. C.; Bruschi, M. L.; Henriques, M.; Negri, M.; Silva, S. \& Svidzinski, T. I. Propolis: a potential natural product to fight Candida species infections. Future Microbiol., 11:1035-46, 2016.

Tobudic, S.; Kratzer, C.; Lassnigg, A. \& Presterl, E. Antifungal susceptibility of Candida albicans in biofilms. Mycoses, 55(3):199204, 2012.

Williams, D. \& Lewis, M. Pathogenesis and treatment of oral candidosis. J. Oral Microbiol., 3:5771, 2011.
Direccion para correspondencia:

Prof. Dr. Ziyad S. Haidar,

DDS, Cert Implantol., MSc OMFS, FRCS(Canada), FACS, MBA, PhD.

Professor and Scientific Director

Faculty of Dentistry

Universidad de Los Andes

Mons. Álvaro del Portillo 12.455

Las Condes

Santiago

CHILE

E-mail: zhaidar@uandes.cl

Recibido : 12-05-2017

Aceptado: 30-06-2017 